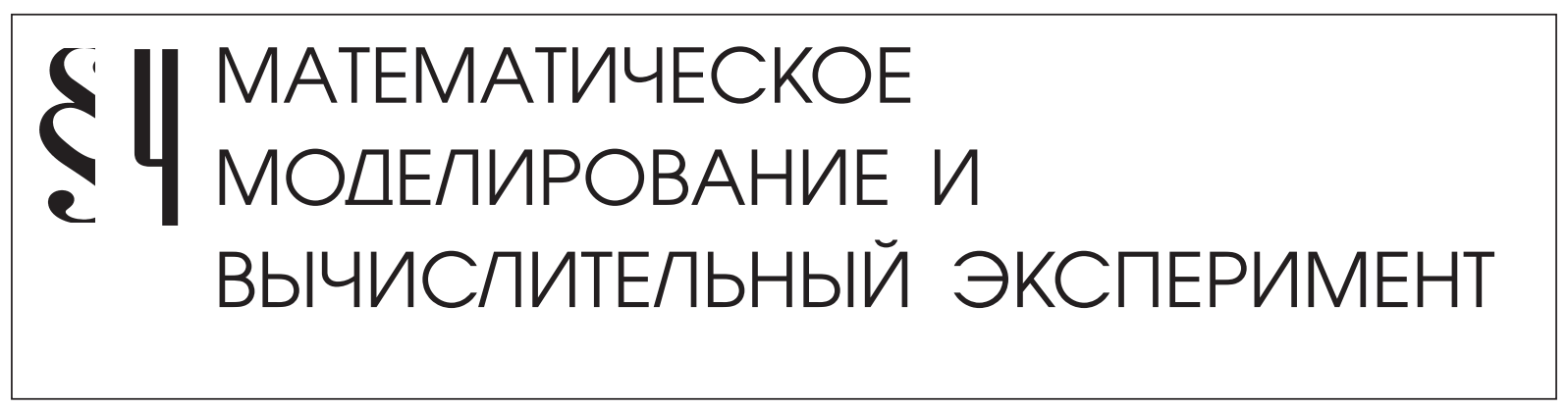

Миронова М.М., Кулифеев Ю.Б.

\title{
ПРОГРАММНЫЙ КОМПЛЕКС МОДЕЛИРОВАНИЯ РЕЖИМОВ УПРАВЛЕНИЯ ГОРИЗОНТАЛЬНЫМ ПОЛЕТОМ БЕСПИЛОТНОГО ЛЕТАТЕЛЬНОГО АППАРАТА
}

Аннотация: Предметом исследования является оптимизация управления скоростью полета и высотой полета летательных аппаратов, реализующего координированное отклонение органов управления с помощью специального алгоритмического обеспечения для пилотажно-навигачионных систем автоматического управления. Решалась задача создания программного комплекса моделирования режимов управления горизонтальным полетом беспилотного летательного аппарата, позволяющего валидизировать (комплексно исследовать для широкого класса условий, режимов и ограничений) алгоритмы управления высотой и скоростью полета с помощью интегрированной системы управления. Полученные результаты представляются применительно к алгоритму адаптивного управления скоростью и высотой полета беспилотного летательного аппарата самолетного типа, обладающему универсальностью. Методология исследования включает методы математического моделирования, управления сложными системами, обратных задач динамики, системного моделирования, адаптивного управления, динамики полета, аэродинамики. Основным результатом исследования является созданный программный комплекс моделирования режимов управления горизонтальным полетом беспилотного летательного апnарата, с помощью которого валидизирован алгоритм управления горизонтальным полетом беспилотного летательного аппарата, обеспечивающий увеличение быстродействия контуров управления скоростью и высотой полета, уменьшая расход топлива, обусловленный стремлением текущей скорости приблизиться к оптимальному значению, за счет вывода летательного аппарата на оптимальную высоту полета. Ключевые слова: моделирование полета, алгоритм управления полетом, управление полетом, беспилотный летательный аппарат, пилотажно-навигационная система, 
продольный канал управления, автомат тяги, быстродействие контура управления, обратная задача динамики, моделирующий программный комплекс

Abstract: The subject of the study is to optimize the management of flight speed and altitude of the aircraft, implementing a coordinated deflection of controls using special algorithmic software for flight control and navigation systems of automatic control. The authors solve the problem of creating a simulation software for controlling the regimes of horizontal flight of an unmanned aircraft, which allows to validate (to carry out a comprehensive study for a wide range of conditions, conditions and restrictions) control algorithms altitude and airspeed with the help of an integrated management system. The results are presented in relation to the adaptive control algorithm speed and altitude UAV aircraft type having versatility. The research methodology includes methods of mathematical modeling, management of complex systems, inverse problems of dynamics, system simulation, adaptive control, flight dynamics, aerodynamics. The main result of the study is a program complex of modeling management regimes of horizontal flight of an unmanned aircraft, which has been used to validated control algorithm level flight of an unmanned aircraft, providing an increase in the speed control loop speed and altitude, reducing fuel consumption, due to the desire of the current rate closer to the optimal value due to the withdrawal of the aircraft at the optimum altitude.

Keywords: speed control loop, autothrottle, longitudinal control channel, flight control and navigation system, unmanned aerial vehicle, flight control, flight control algorithm, flight simulation, the inverse problem of dynamics, modeling software package

Одной из актуальных задач в авиации является оптимизация управления скоростью полета и высотой полета летательных аппаратов [1-3]. Наибольшую сложность при этом имеет обеспечение согласованности функционирования системы «летательный аппарат - силовая установка (СУ)» в рамках концепции интегрированного управления [4-6]. Для максимально полного использования летно-технических возможностей самолета необходимо координированное отклонение органов управления, а это, в свою очередь, предусматривает совершенствование алгоритмического обеспечения для пилотажнонавигационных систем автоматического управления (САУ) [7-12].

Для решения названных задач разработан программный комплекс моделирования режимов управления горизонтальным полетом беспилотного летательного аппарата (БЛА). В статье представлены алгоритмы автоматического управления в продольном канале на этапе горизонтального полета. Предлагается адаптивный алгоритм управления скоростью и высотой полета беспилотного летательного аппарата самолетного типа, обладающий универсальностью, применимый для широкого перечня самолетов и СУ (рис. 1).

\section{Постановка задачи}

Алгоритм управления скоростью и высотой полета реализует регулятор, выводящий 
БЛА на заданную скорость и высоту полета и поддерживающий их постоянными, воздействуя на секторы газа двигателей. Основная цель математического моделирования алгоритма управления работы состояла в решении следующих задач:

- определение возможностей улучшения полетных характеристик БЛА на основе использования метода обратных задач динамики;

- $\quad$ определение облика пилотажно-навигационной САУ в продольном движении на этапе горизонтального полета БЛА;

- $\quad$ разработка алгоритмов автоматического управления в продольном канале на этапе горизонтального полета с целью улучшения полетных характеристик в условиях атмосферной нестабильности;

- исследование эффективности предлагаемых алгоритмов для пилотажно-навигационной САУ при различных условиях полета.

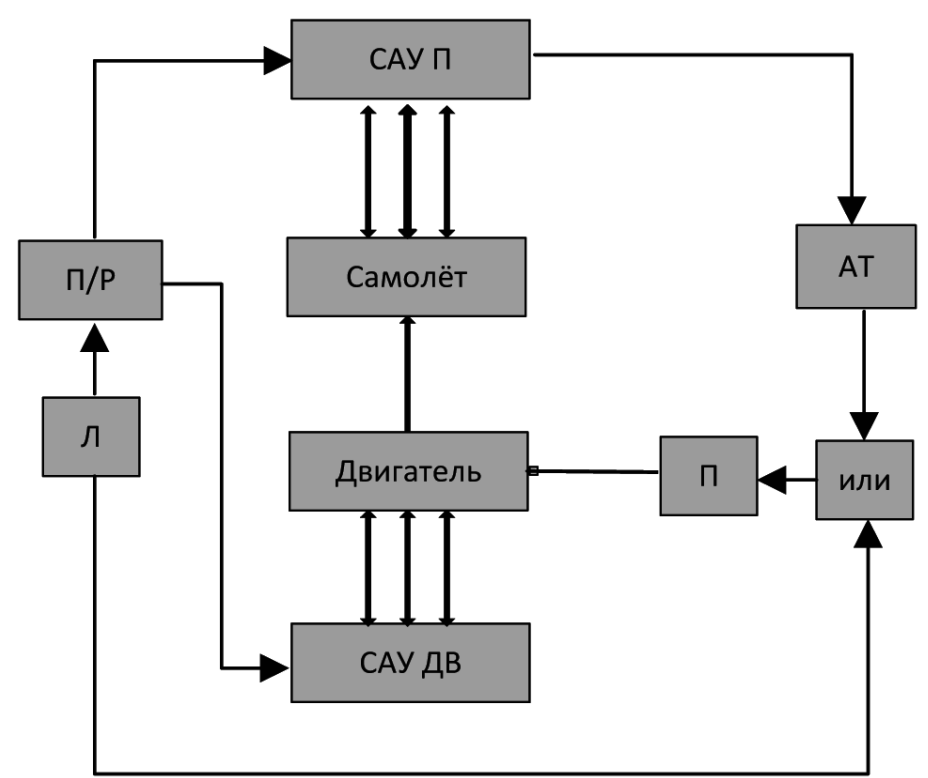

Рисунок 1 - Структурная схема интегрированной системы управления скоростью полета: ДВ - дВигатель; САУ ДВ - САУ двигателя; САУП - система управления полетом; Л - летчик; П/Р - пульт режимов; П - привод РУД; АТ - автомат тяги.

В ходе исследований получены следующие основные результаты:

- $\quad$ математическая модель пространственного наземно-воздушного движения БЛА на этапе горизонтального полета;

- $\quad$ алгоритм автоматического управления в продольном канале на этапе горизонтального полета БЛА;

Разработанные алгоритмы имеют двойное назначение, а именно могут использоваться в помощь летчику при горизонтальном полете, так и в обеспечении горизонтального полета БЛА самолетного типа.

Описание работы алгоритма начнем со структурной схемы интегрированной системы 
управления скоростью и высотой полета, которая наглядно показывает принцип работы комплекса разработанных алгоритмов (рис. 2).

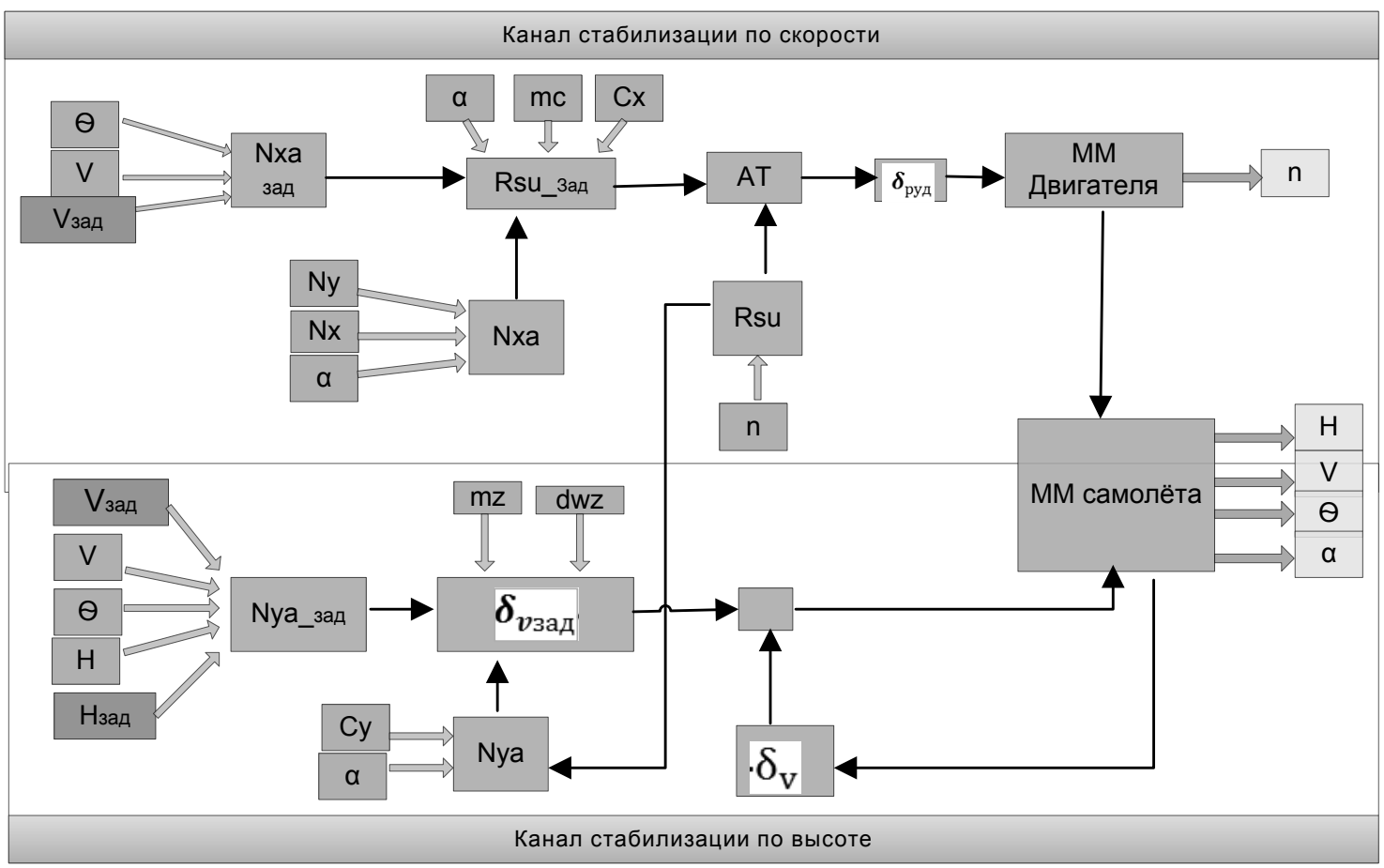

Рисунок 2 - Структура комплекса алгоритмов системы отработки заданного маневра БЛА по высоте и скорости полета

Вывод алгоритма управления скоростью и высотой полета основывается на математической модели объекта, объединяющей аэродинамическую модель, динамическую модель и модель ветровых возмущений [13-20]. Поэтому прежде чем перейти к описанию работы самого алгоритма, опишем применяемые базовые модели.

Структура и точность математической модели определяется характером задач, для решения которых она предназначена. Наибольшая точность требуется, если модель используется не только для решения задачи управления, но и для расчета тяговых, экономических и высотно-скоростных характеристик двигателя. В нашем случае - это задача управления интегрированной системой «ЛА - СУ».

Пусть динамика изменения скорости полета БЛА в скоростной системе координат для продольной плоскости определяется системой нелинейных дифференциальных уравнений:

$$
\begin{aligned}
& \dot{V}(t)=\left[k_{1}(\bar{n}) \bar{n}(t) \cos \left(\alpha+\alpha_{\text {дв }}\right)-C_{x} S q-G \sin \theta\right] / m, \\
& \dot{\bar{n}}(t)=a\left(k \delta_{\text {руд }}-\bar{n}(t)\right)+b\left(k \delta_{\text {руд }}-\bar{n}(t)\right)^{2},
\end{aligned}
$$

где $m$ - масса самолета; $G$ - его вес; $S$ - площадь крыла в плане; $C_{x}$ - коэффициент лобового сопротивления; $q$ - скоростной напор; $\alpha$ - угол атаки; $\alpha_{\text {дв }}$-угол установки двигателя; 
$\theta$ - угол наклона траектории; $a, b, k$ - параметры модели силовой установки; $\bar{n}(t)$ - относительное значение частоты вращения компрессора двигателя, определяемое выражением:

$$
\bar{n}(t)=\frac{n(t)}{n_{\max }}
$$

здесь $k_{1}(\bar{n})$ - коэффициент (в общем случае полином), характеризующий связь между частотой вращения компрессора и силой тяги при фиксированных значениях параметров полета и внешних условий [21].

Следует отметить, что в математической модели двигателя (1) заданное значение частоты вращения компрессора $n_{\text {кз }}$ задается программой системы регулирования двигателя и является функцией внешних условий и внутренних параметров силовой установки (частоты вращения вентилятора, температуры за турбиной, перемещения $\delta_{\text {руд }}$ и т.д.). Здесь же для простоты полагается, что: $n_{\text {кз }}=k \delta_{\text {руд }}$

\section{Алгоритм управления горизонтальным полетом беспилотного летательного аппарата}

Для задачи управления скоростью полета функцию рассогласования выбираем в виде:

$$
F(V)=V(t)-V_{3}(t)
$$

Требуется найти такое управление $\delta_{\text {руд }}(t)$, которое обеспечивает движение БЛА со скоростью полета, равной $V_{3}(t)$. Учитывая аналитическую связь между $\delta_{\text {руд }}(t)$ и рассогласованием $F(V)$, примем $n=2$. Тогда уравнение (2) для функции $F(V)$ примет вид:

$$
F^{(n)}(x)+\lambda_{n-1} F^{(n-1)}(x)+\ldots+\lambda_{0} F(x)=0 .
$$

В связи с тем, что у различных типов ЛА существуют и различные штатные системы автоматического управления, связывающие отклонения рулевых поверхностей с выходными координатами, то и решение (3) будем выполнять в несколько этапов [12, 13, 22-24]. Такой подход возможен, если параметры $\lambda_{1}$ и $\lambda_{0}$ выбрать так, чтобы решение (3) стремилось к нулю по некоторой комбинации экспоненциальных законов.

Первый этап синтеза заключается в определении заданного значения продольной и

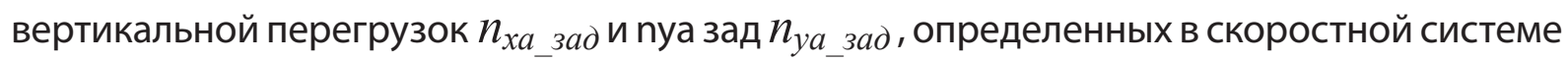
координат.

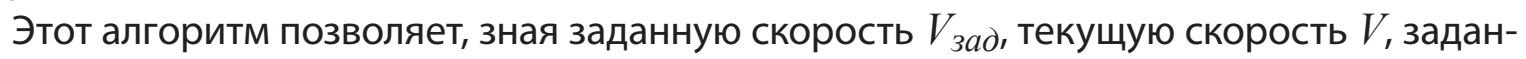

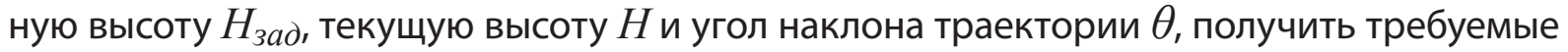

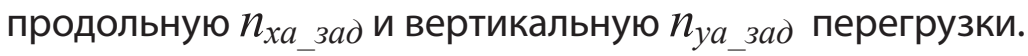

Рассмотрим методику вычисления заданного значения продольной перегрузки $n_{x a \_z a d .}$

Как известно, движение самолета в скоростной системе координат в продольном 
канале описывается уравнениями вида $[13,14]$ :

$$
\left.\begin{array}{l}
\dot{V}=g \cdot\left(n_{x a}-\sin \theta\right) \\
\dot{\theta}=\frac{g \cdot\left(n_{y a}-\cos \theta\right)}{V} \\
\dot{H}=V \cdot \sin \theta
\end{array}\right\}
$$

Пусть $\varepsilon=V-V_{\text {зад }}$, где $\varepsilon$ - отклонение текущей скорости самолета от заданной скорости.

В соответствии с методом обратных задач динамики потребуем, чтобы выполнялось условие, заданное дифференциальным уравнением:

$$
\dot{\varepsilon}+\lambda_{0 v} \varepsilon=0
$$

тогда после подстановки имеем:

$$
\left(\dot{V}-\dot{V}_{\text {зад }}\right)+\lambda_{0 V}\left(V-V_{\text {зад }}\right)=0
$$

Заменив в уравнении (5) производную текущей скорости правой частью первого уравнения системы (4), получим:

$$
g\left(n_{x a}-\sin \theta\right)+\lambda_{0 V}\left(V-V_{\text {зад }}\right)=\dot{V}_{\text {зад }}
$$

Поскольку требуемый режим горизонтального полета является установившимся режимом, то полагая в (6) $V_{\text {зад }}=0$, получим соотношение, определяющее заданную перегрузку:

$$
n_{x a_{-} \text {зад }}=\sin \theta-\frac{\lambda_{0 V}}{g}\left(V-V_{\text {зад }}\right)
$$

Методика вывода соотношения, определяющего заданное значение вертикальной перегрузки $n_{y a}$ зад , аналогична вышеизложенной.

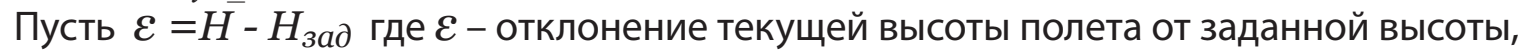

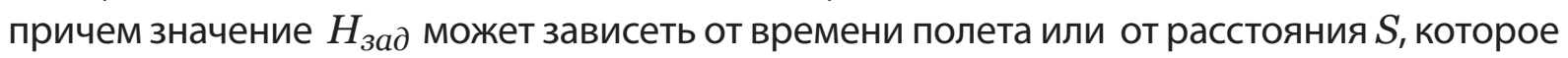
пролетел самолет с некоторого момента времени.

Потребуем выполнения условия $\ddot{\varepsilon}+\lambda_{1 H} \dot{\varepsilon}+\lambda_{0 H} \varepsilon=0$, тогда

$$
\left(\ddot{H}-\ddot{H}_{\text {зад }}\right)+\lambda_{1 н}\left(\dot{H}-\dot{H}_{\text {зад }}\right)+\lambda_{0_{H}}\left(H-H_{\text {зад }}\right)=0 .
$$


Продифференцировав третье уравнение системы (4) по времени получим:

$$
\ddot{H}=d(V \sin \theta) / d t=\dot{V} \sin \theta+V \cos \theta \dot{\theta}
$$

Учитывая, что из формулы (5) при условии $\dot{V}_{\text {зад }}=0$, следует:

$$
\dot{V}=-\lambda_{0 V}\left(V-V_{3 a d}\right)
$$

а из второго уравнения системы (4) имеем $V=\frac{g\left(n_{y a}-\cos \theta\right)}{\cdot}$ получим выражение вида:

$$
\begin{aligned}
& \left(-\lambda_{0 H}\left(V-V_{\text {зад }}\right) \sin \theta+g \cos \theta\left(n_{y a}-\cos \theta\right)-\ddot{H}_{\text {зад }}\right)+ \\
& +\lambda_{1 H}\left(V \sin \theta-\dot{H}_{\text {зад }}\right)+\lambda_{0 H}\left(H-H_{\text {зад }}\right)=0 .
\end{aligned}
$$

Из последнего выражения определяется заданная вертикальная перегрузка:

$n_{y a_{-} \text {зад }}=\cos \theta-\frac{1}{g}\left(\left(\lambda_{1 H} V-\lambda_{0 V}\left(V-V_{\text {зад }}\right)\right) \operatorname{tg} \theta+\frac{\lambda_{0 H}\left(H-H_{\text {зад }}\right)-\ddot{H}_{\text {зад }}-\lambda_{1 H} \dot{H}_{\text {зад }}}{\cos \theta}\right)$

Для выбранного БЛА коэффициенты в контурах регулирования скорости и высоты полета были выбраны следующими значениями:

$$
\lambda_{0 V}=1 / T_{V}
$$

где $T_{v}=30[\mathrm{c}]$.

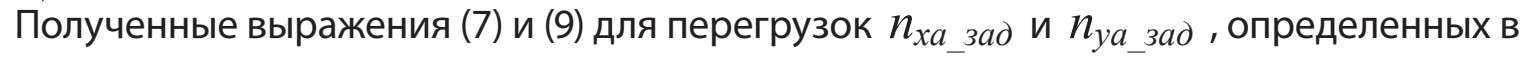
скоростной системе координат, используются далее для определения заданных значений угла отклонения руля высоты и тяги силовой установки.

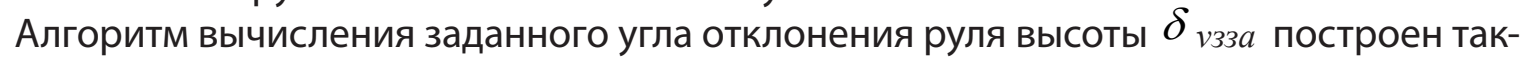
же на основе метода обратных задач динамики [15]. Этот алгоритм реализует закон управления в контуре вертикальной перегрузки.

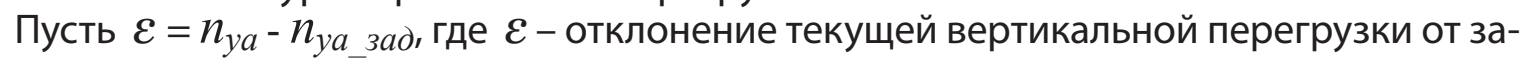
данной. Потребуем, чтобы выполнялось условие:

$$
\dot{\varepsilon}+\lambda_{1 n} \dot{\varepsilon}+\lambda_{0 n} \varepsilon=0
$$

Тогда

$$
\left(\ddot{n}_{y a}-\ddot{n}_{y a_{-} \text {зад }}\right)+\lambda_{1 n}\left(\dot{n}_{y a}-\dot{n}_{y a_{-} \text {зад }}\right)+\lambda_{0 n}\left(n_{y a}-n_{y a_{-} \text {зад }}\right)=0
$$


Примем допущение, что в течение времени дискретности работы алгоритма

$$
\ddot{n}_{y a_{-} \text {заd }}=\dot{n}_{y a_{-} 3 a d}=0
$$

Найдем первую и вторую производные вертикальной перегрузки $n_{y a}$ :

$$
\begin{aligned}
& \dot{n}_{y a}=\frac{R_{c y}}{G}\left[\cos \left(\alpha+\varphi_{\partial в}\right)+\frac{C_{y}^{\alpha} q S_{\kappa p}}{R_{c y}}\right] \dot{\alpha} \\
& \ddot{n}_{y a}=\frac{R_{c y}}{G}\left[-\sin \left(\alpha+\varphi_{\partial в}\right) \dot{\alpha}^{2}+\left(\cos \left(\alpha+\varphi_{\partial в}\right)+\frac{C_{y}^{\alpha} q S_{\kappa p}}{R_{c y}}\right) \ddot{\alpha}\right] \\
& \alpha=\vartheta-\theta \\
& \dot{\alpha}=\omega_{z}-\dot{\theta}=\omega_{z}-\frac{g}{V}\left(n_{y a}-\cos \theta\right) .
\end{aligned}
$$

Для упрощения вычислений в силу малости величины $\ddot{\theta}$ в сравнении с величиной $\dot{\omega}_{z}$ сделаем допущение:

$$
\ddot{\alpha}=\dot{\omega}_{z}-\ddot{\theta}=\dot{\omega}_{z}
$$

В таком случае выражение для определения второй производной вертикальной перегрузки будет иметь следующий вид:

$$
\ddot{n}_{y a}=\frac{R_{c y}}{G}\left[-\sin \left(\alpha+\varphi_{\partial в}\right) \dot{\alpha}^{2}+\left(\cos \left(\alpha+\varphi_{\partial b}\right)+\frac{C_{y}^{\alpha} q S_{\kappa p}}{R_{c y}}\right) \dot{\omega}_{z}\right]
$$

Тогда из формулы (12) получим выражение для углового ускорения $\dot{\omega}_{z}$ при вращении самолета вокруг оси $\mathrm{Oz}$ :

$$
\dot{\omega}_{z}=\frac{\frac{G_{c y}}{R_{y a}}+\sin \left(\alpha+\varphi_{\partial в}\right) \dot{\alpha}^{2}}{\cos \left(\alpha+\varphi_{\partial в}\right)+\frac{C_{y}^{\alpha} q S_{\kappa p}}{R_{c y}}} .
$$

В формулу будем подставлять значение второй производной вертикальной перегрузки из условия (12), т. е. здесь

$$
\ddot{n}_{y a}=-\lambda_{1 n} \dot{n}_{y a}-\lambda_{0 n}\left(n_{y a}-n_{y a_{-} 3 a \partial}\right) .
$$


Коэффициент продольного аэродинамического момента связан с угловым ускорением $\dot{\omega}_{z}$ соотношением:

$$
m_{z}=\frac{J \dot{\omega}_{z}+y_{\partial b} R_{c y}}{q S_{\kappa p} b_{a}}
$$

где $y_{\partial в}$ - величина, значение которой равно длине перпендикуляра, опущенного из центра масс самолета на вектор тяги, а знак определяется положением двигателя относительно центра масс самолета. Если двигатель находится ниже центра масс самолета, то $y_{\partial \beta}<0$.

С другой стороны, поскольку

$$
m_{z}=m_{z 0}+m_{z}^{\alpha} \delta_{v 3 s a}+m_{z}^{\bar{w}_{z}} \bar{w}_{z},
$$

заданный угол отклонения руля высоты будет определяться соотношением:

$$
\delta_{v 33 a}=\frac{m_{z}^{-}\left(m_{z 0}+m_{z}^{\alpha} \alpha+m_{z}^{\overline{\mathcal{W}}_{z}} \frac{\bar{W}_{z}}{\left.\frac{b a}{V}\right)}\right.}{m_{z}^{\wedge V}}
$$

Таким образом, порядок вычисления заданного значения угла отклонения руля высоты сводится к последовательному расчету по следующим формулам:

$$
\begin{aligned}
& \delta_{v 33 a}=\frac{m_{z}-\left(m_{z 0}+m_{z}^{\alpha} \alpha+m_{z}^{\bar{w}_{z}} \overline{\mathcal{W}}_{z} \frac{b a}{V}\right)}{m_{z}^{\wedge V}} \\
& m_{z}=\frac{J \dot{\omega}_{z}+y_{\partial \beta} R_{c y}}{q S_{\kappa p} b_{a}} \\
& \frac{G}{R} \ddot{n}_{y a}+\sin \left(\alpha+\varphi_{\partial в}\right) \dot{\alpha}^{2} \\
& \dot{\omega}_{z}=\frac{c y}{\cos \left(\alpha+\varphi_{\partial в}\right)+\frac{C_{y}^{\alpha} q S_{\kappa p}}{R_{c y}}} \\
& \ddot{n}_{y a}=-\lambda_{1 n} \dot{n}_{y a}-\lambda_{0 n}\left(n_{y a}-n_{y a_{-} \text {заd }}\right) \\
& \dot{n}_{y a}=\frac{R_{c y}}{G}\left[\cos \left(\alpha+\varphi_{\partial в}\right)+\frac{C_{y}^{\alpha} q S_{\kappa p}}{R_{c y}}\right] \dot{\alpha} \\
& \dot{\alpha}=\omega_{z}-\dot{\theta}=\omega_{z}-\frac{g}{V}\left(n_{y a}-\cos \theta\right) \text {. }
\end{aligned}
$$

Заметим, что система уравнений (15) решается в порядке обратном записи этих 
уравнений выше

Вычисление заданной тяги силовой установки $R_{c y_{-} \text {зад }}$ осуществляется по формуле:

$$
R_{c y_{-} \text {зад }}=\left(n_{x a_{-} \text {зад }} \cdot m \cdot g+C_{x a} \cdot q \cdot S_{\kappa p}\right) \cdot \frac{1}{\cos \left(\alpha+\varphi_{\partial в}\right)}
$$

где $C_{x a}$ - аэродинамический коэффициент силы лобового сопротивления; $S_{\kappa p}$ - площадь крыла самолета; $\varphi_{\partial в}$ - угол установки двигателей.

\section{Результаты моделирования разработанных алгоритмов}

Исходный режим (балансировочный): $H=10000$ [m], $V=776[\mathrm{~km} / \mathrm{h}], M=0.72$. Новый режим полета: $H=10200[\mathrm{~m}], V=800[\mathrm{~km} / \mathrm{h}]$.

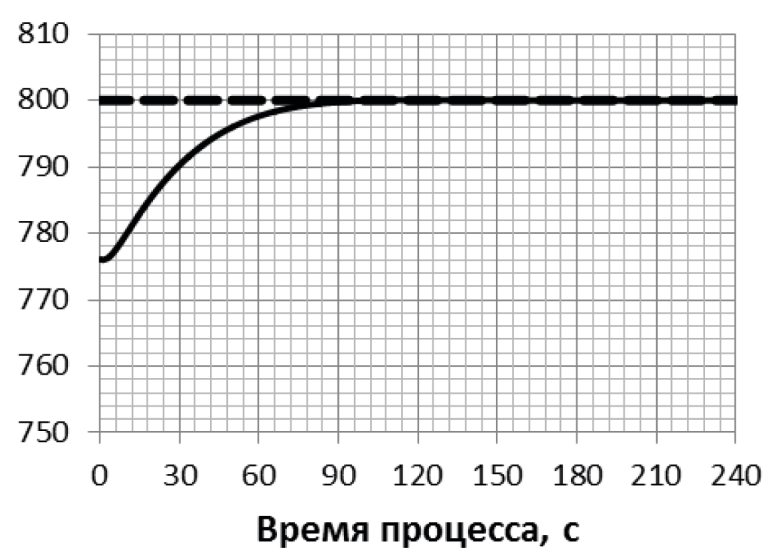

Рисунок 3 - Изменение скорости при смене режима полета (сплошная линия - заданное значение, пунктирная линия рассчитанное значение).

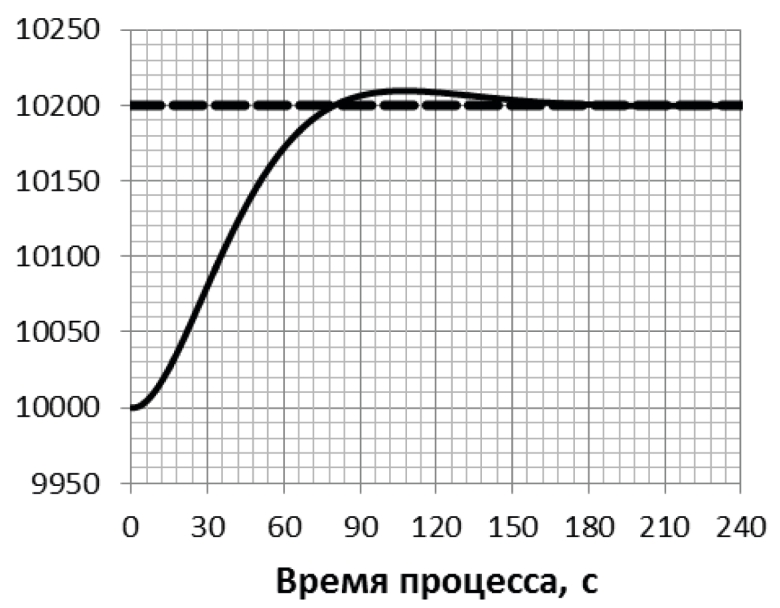

Рисунок 4 - Изменение высоты при смене режима полета (сплошная линия - заданное значение, пунктирная линия рассчитанное значение). 


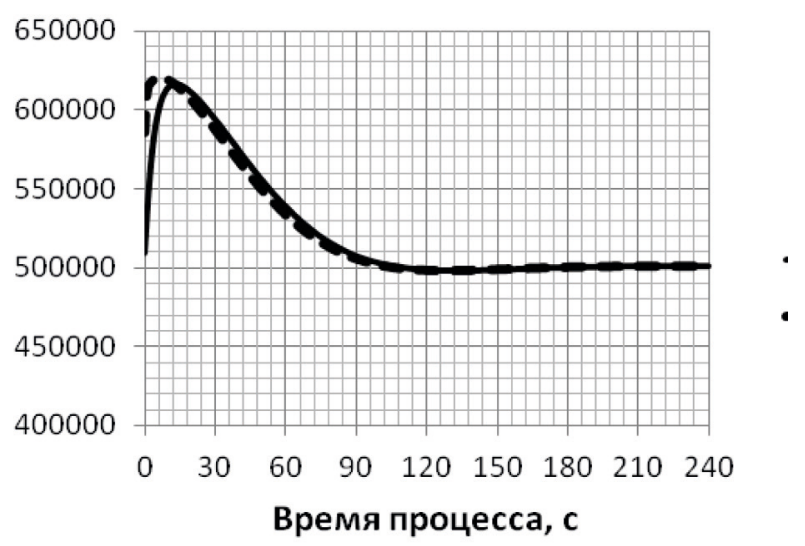

Рисунок 5 - Изменение тяги силовой установки самолета при смене режима полета (сплошная линия - заданное значение, пунктирная линия - рассчитанное значение)

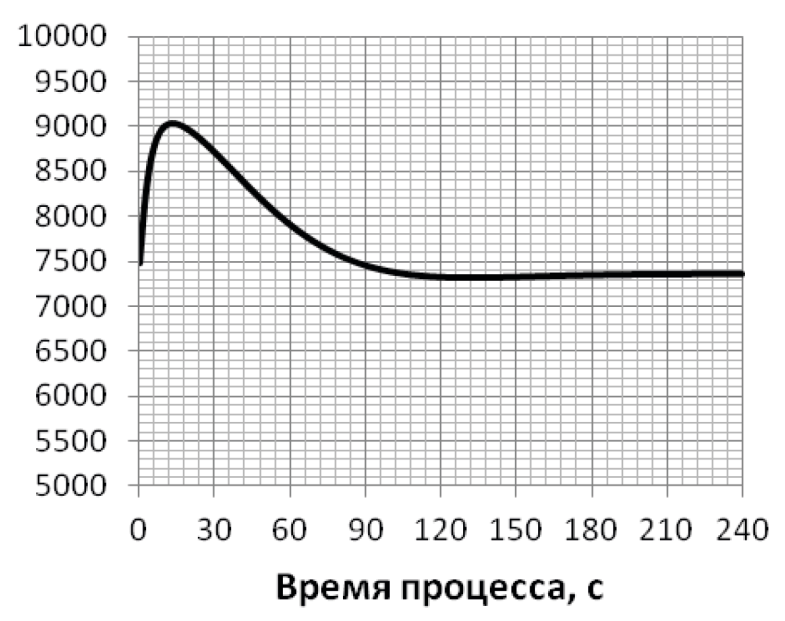

Рисунок 6 - Изменение частоты вращения компрессора высокого давления при смене режима полета

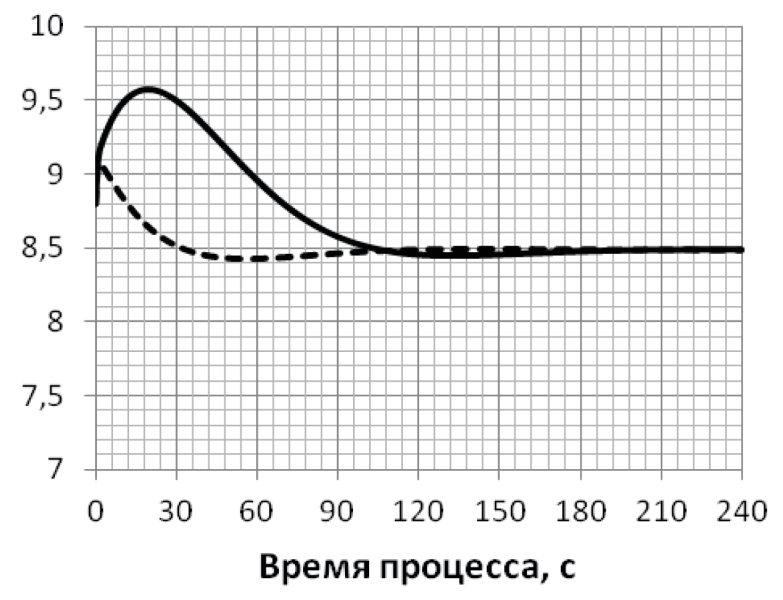

Рисунок 7 - Изменение углов тангажа и атаки при смене режима полета (сплошная линия - заданное значение, пунктирная линия - рассчитанное значение) 


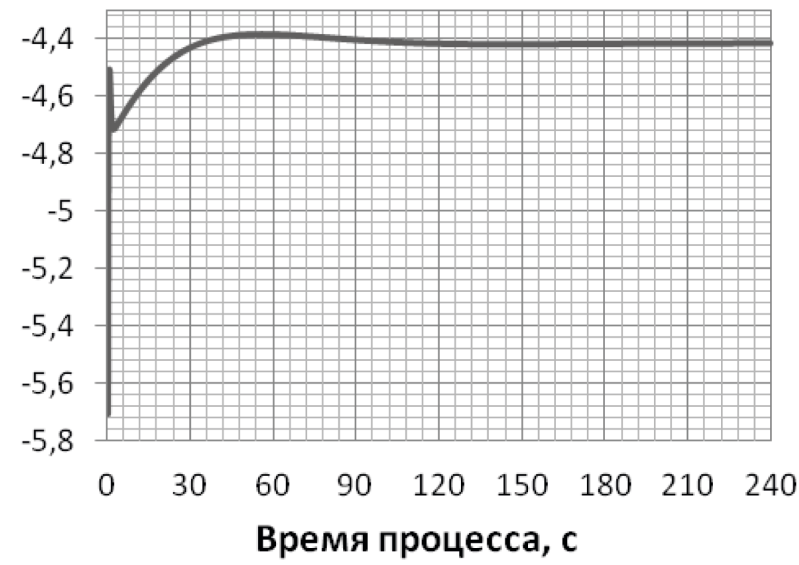

Рисунок 8 - Изменение угла отклонения руля высоты

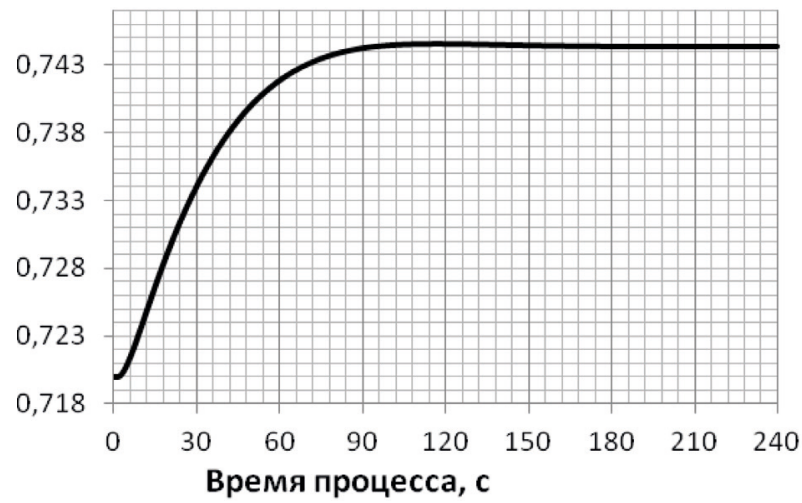

Рисунок 9 - Изменение числа Маха при смене режима полета.

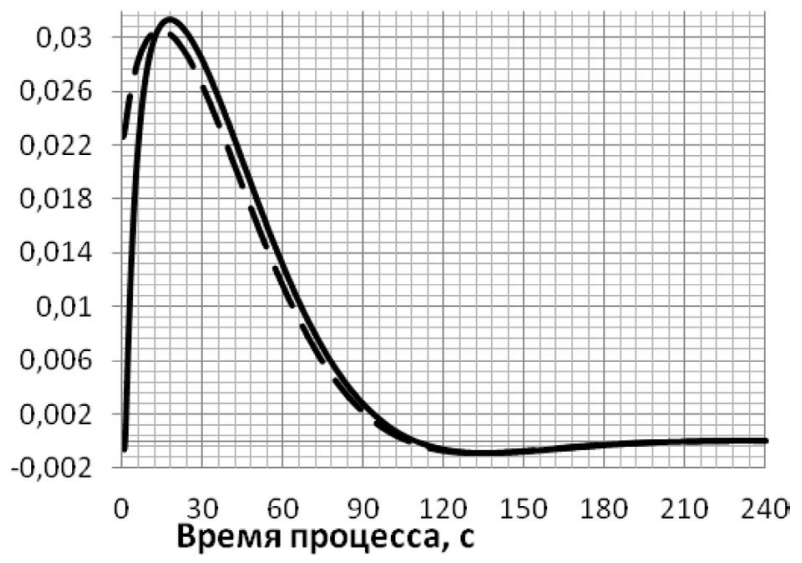

Рисунок 10 - Изменение перегрузки по оси воздушной скорости при смене режима полета (сплошная линия - заданное значение, пунктирная линия - рассчитанное значение) 


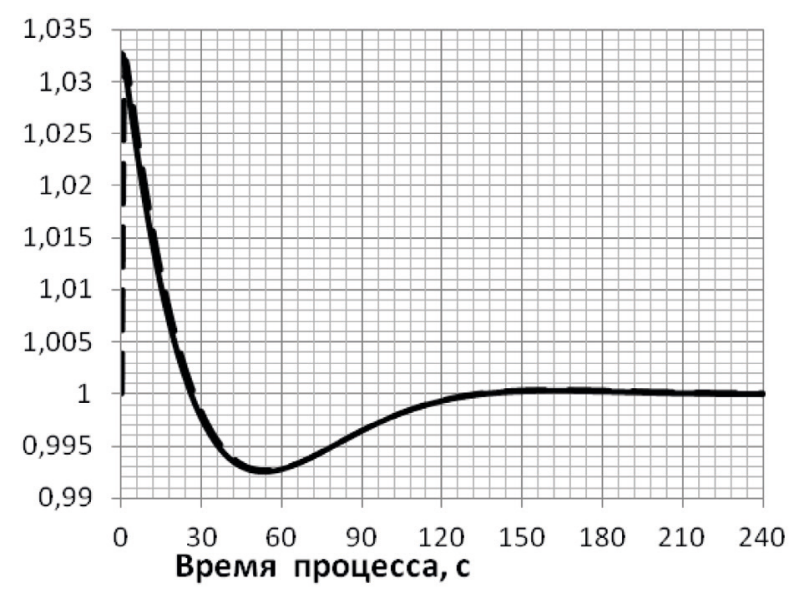

Рисунок 11 - Изменение перегрузки по оси подъемной силы при смене режима полета (сплошная линия - заданное значение, пунктирная линия - рассчитанное значение).

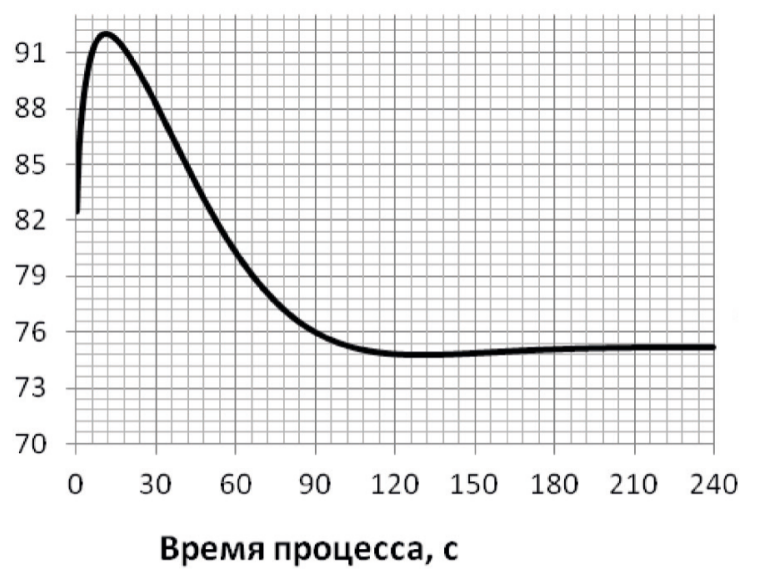

Рисунок 12 - Изменение положения РУД при смене режима полета.

Из представленных графиков (рис. 3 - 12) следует, что:

а) произошел успешный переход беспилотного летательного аппарата по скорости и высоте;

б) изменение тяги двигателя $R$ с ростом скорости полета определяется противоположным изменением величин $R_{y d}$ и $G_{\dot{b}}$.

в) с увеличением скорости полета произошло и увеличение числа Маха.

г) при увеличении скорости полета и высоты перегрузки $n_{x a}$ и $n_{y a}$ сперва увеличивались до 20 секунды, затем монотонно уменьшались, но затем к 100 секунде их значение стабилизировалось и осталось неизменным до конца моделирования.

\section{Дроссельные характеристики двигателя прямой реакции}

Как подтверждение корректности работы алгоритма приведем графики поведения 
тяги во время изменения высоты и скорости полета, а также оборотов двигателя - рисунки $13,14,15$

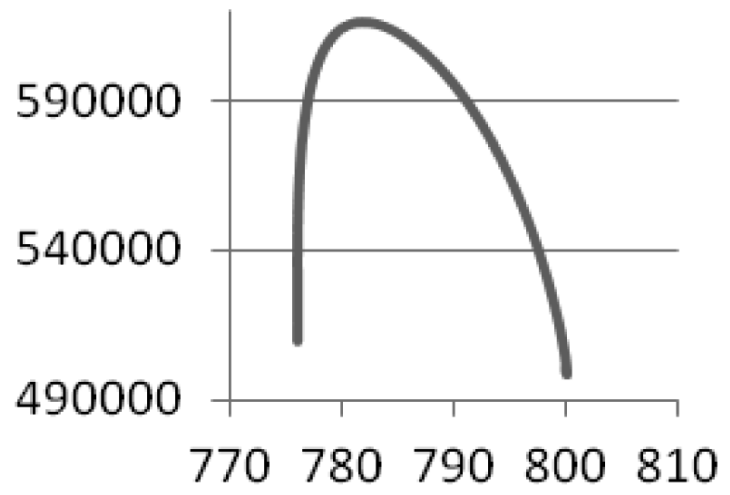

Рисунок 13 - Зависимость тяги от скорости полета

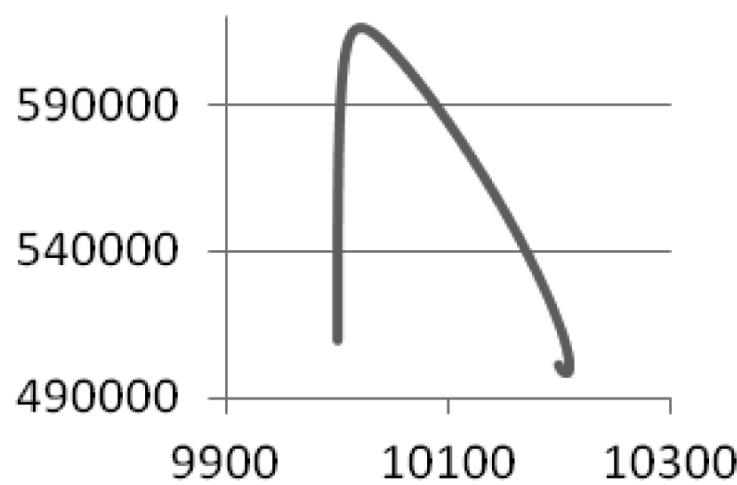

Рисунок 14 - Зависимость тяги от высоты полета

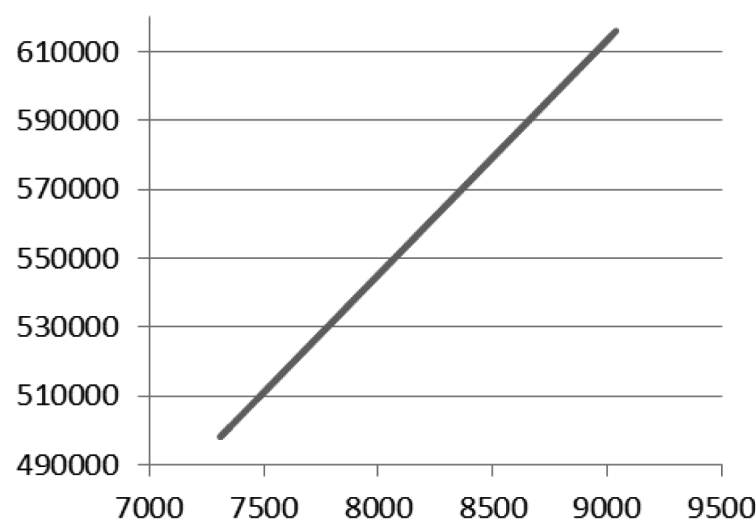

Рисунок 15 - Дроссельные характеристики двигателя Д-18Т при смене режима полета. 
Из характера изменения кривой $R=R(n)$ видно, что темп возрастания тяги сувеличением оборотов также возрастает. Для современных двигателей увеличение оборотов на 1 \% в районе оборотов режима максимальной тяги приводит кувеличению тяги на 3 - $4 \%$.

Отсюда вытекает требование высокой точности поддержания оборотов для обеспечения максимальной тяги двигателя.

Изменение положения РУД при смене режима полета происходило следующим образом: значение увеличивалось до 20 секунды, затем монотонно уменьшалось, но затем к 100 секунде значение стабилизировалось и осталось неизменным до конца моделирования на уровне 74 градуса. Результаты работы алгоритма положительные, корректность алгоритма подтверждается.

$$
\text { * * * }
$$

В ходе исследования решена задача создания программного комплекса моделирования режимов управления горизонтальным полетом беспилотного летательного аппарата, позволяющего валидизировать (комплексно исследовать для широкого класса условий, режимов и ограничений) алгоритмы управления высотой и скоростью полета с помощью интегрированной системы управления. С помощью разработанного программного комплекса валидизирован алгоритм управления горизонтальным полетом беспилотного летательного аппарата, который увеличивает быстродействие контуров управления скоростью и высотой полета, уменьшая расход топлива, обусловленный стремлением текущей скорости приблизиться к оптимальному значению, за счет вывода летательного аппарата на оптимальную высоту полета.

\section{Библиография :}

1. Харьков В.П., Халютин С.П. Управление вектором скорости полета летательного аппарата на основе энергетического подхода // Научный вестник Московского государственного технического университета гражданской авиации. 2015. № 213 (3). С. 73-80.

2. Шибанов Г.П. Оптимизация систем управления летательным аппаратом по критериям управляемости и наблюдаемости // Мехатроника, автоматизация, управление. 2012. № 4. С. 57-61.

3. Гулевич С.П., Александровский Б.В., Веселов Ю.Г. Обоснование основных требований к характеристикам движения беспилотных летательных аппаратов двойного назначения // Проблемы безопасности полетов. 2008. № 8. C. 25-39.

4. Северцев Н.А., Шипилов В.В. Дистанционно управляемые беспилотные двухсредные аппараты // Вопросы теории безопасности и устойчивости систем. 2014. № 16. С. 107-116.

5. Харьков В.П. Алгоритм управления относительным положением беспилотных летательных аппаратов // Информационно-измерительные и управляющие системы. 2015. Т. 13. № 5. С. 33-40. 
6. Гулевич С.П., Веселов Ю.Г. Выбор и обоснование минимально необходимого объема параметров полетной информации, измеряемых на борту беспилотного летательного аппарата // Проблемы безопасности полетов. 2008. № 11. С. 58-63.

7. Буков В.Н., Бочаров А.С., Сельвесюк Н.И. Синтез контуров управления летательного аппарата с гарантированной точностью // Гироскопия и навигация. 2006. № 3 (54). С. 86.

8. Кулифеев Ю.Б., Афанасьев Ю.Н. Алгоритм автоматического приведения самолета к заданным высоте и скорости горизонтального полета // Мехатроника, автоматизация, управление. 2011. № 9. С. 54-58.

9. Макаренко В.Г., Подорожняк А.А., Рудаков С.В., Богомолов А.В. Инерциально-спутниковая навигационная система управления транспортными средствами // Проблемы управления. 2007. № 1. С. 64-71.

10. Савченко А.Ю., Сельвесюк Н.И. Разработка методического аппарата синтеза робастных децентрализованных законов управления с модельной координацией // Информатика и системы управления. 2014. № 2 (40). C. 158-167.

11. Гулевич С.П., Веселов Ю.Г. Методика определения коэффициента момента тангажа беспилотных летательных аппаратов по материалам натурных экспериментов // Проблемы безопасности полетов. 2009. № 11. С. 47-52.

12. Харьков В.П., Михалев И.С. Адаптивный алгоритм управления скоростью полета летательного аппарата // Информационно-измерительные и управляющие системы. 2011. Т. 9. № 1. С. 9-15.

13. Кулифеев Ю.Б. Определение дискретных математических моделей динамических объектов, заданных линейными дифференциальными уравнениями с постоянными коэффициентами // Информационноизмерительные и управляющие системы. 2008. Т. 6. № 11. С. 5-19.

14. Кулифеев Ю.Б., Лихачев И.В. Синтез алгоритма управления бесконтактным вентильным двигателем методом обратных задач динамики // Мехатроника, автоматизация, управление. 2007. № 1. С. 28-36.

15. Харьков В.П., Корсун 0.Н., Гребнев О.Н. Синтез управления беспилотным летательным аппаратом на основе метода обратных задач динамики и параметрической идентификации // Вестник компьютерных и информационных технологий. 2010. № 12. С. 18-23.

16. Кулифеев Ю.Б., Афанасьев Ю.Н. Алгоритмизация автоматической посадки самолета в условиях метеоминимума // Мехатроника, автоматизация, управление. 2012. № 11. С. 60-66.

17. Гулевич С.П., Веселов Ю.Г. Метод определения скорости приземления беспилотных летательных аппаратов на основе результатов математического моделирования и натурных экспериментов // Проблемы безопасности полетов. 2008. № 6. С. 33-37.

18. Макаренко В.Г., Богомолов А.В., Рудаков С.В., Подорожняк А.А. Технология построения инерциальноспутниковой навигационной системы управлениятранспортными средствами снейросетевой оптимизацией состава вектора измерений // Мехатроника, автоматизация, управление. 2007. № 1. С. 39-44.

19. Кулифеев Ю.Б., Алексеев Э.О. Алгоритм отработки заданной траектории движения самолета по взлетнопосадочной полосе // Мехатроника, автоматизация, управление. 2007. № 10. С. 73-78.

20. Харьков В.П. Синтез оптимального управление нелинейными динамическими системами на основе концепции обратных задач динамики // Труды международного симпозиума Надежность и качество. 2012. T. 1. C. 491-492.

21. Гуревич О.С. Гольберг Ф.Д., Селиванов О.Д. Интегрированное управление силовой установкой многорежимного самолета. М.: Машиностроение, 1994. 304 с. 
22. Коломиец Л.В., Федоров М.В., Богомолов А.В., Мережко А.Н., Солдатов А.С., Есев А.А. Метод поддержки принятия решений по управлению ресурсами при испытаниях авиационной техники // Информационноизмерительные и управляющие системы. 2010. Т. 8. № 5. С. 38-40.

23. Рудаков И.С., Рудаков С.В., Богомолов А.В. Методика идентификации вида закона распределения параметров при проведения контроля состояния сложных систем // Информационно-измерительные и управляющие системы. 2007. Т. 5. № 1. С. 66-72.

24. Еремин Е.М., Русскин А.В. Способ расчета оценки максимально допустимой взлетной массы беспилотного летательного аппарата на этапе его проектирования // Оборонный комплекс-научно-техническому прогрессу России. 2014. № 1 (121). С. 39-42.

25. Байкин В.А., Стецюк А.Н. Моделирование информационного управления функционированием системы космической связи и навигации // Тренды и управление. - 2015. - 2. - C. 162 - 166. DOI: 10.7256/23079118.2015.2.14408.

\section{References:}

1. Khar'kov V.P., Khalyutin S.P. Upravlenie vektorom skorosti poleta letatel'nogo apparata na osnove energeticheskogo podkhoda // Nauchnyi vestnik Moskovskogo gosudarstvennogo tekhnicheskogo universiteta grazhdanskoi aviatsii. 2015. № 213 (3). S. 73-80.

2. Shibanov G.P. Optimizatsiya sistem upravleniya letatel'nym apparatom po kriteriyam upravlyaemosti i nablyudaemosti // Mekhatronika, avtomatizatsiya, upravlenie. 2012. № 4. S. 57-61.

3. Gulevich S.P., Aleksandrovskii B.V., Veselov Yu.G. Obosnovanie osnovnykh trebovanii k kharakteristikam dvizheniya bespilotnykh letatel'nykh apparatov dvoinogo naznacheniya // Problemy bezopasnosti poletov. 2008. № 8. S. 25-39.

4. Severtsev N.A., Shipilov V.V. Distantsionno upravlyaemye bespilotnye dvukhsrednye apparaty // Voprosy teorii bezopasnosti i ustoichivosti sistem. 2014. № 16. S. 107-116.

5. Khar'kov V.P. Algoritm upravleniya otnositel'nym polozheniem bespilotnykh letatel'nykh apparatov // Informatsionno-izmeritel'nye i upravlyayushchie sistemy. 2015. T. 13. № 5. S. 33-40.

6. Gulevich S.P., Veselov Yu.G. Vybor i obosnovanie minimal'no neobkhodimogo ob"ema parametrov poletnoi informatsii, izmeryaemykh na bortu bespilotnogo letatel'nogo apparata // Problemy bezopasnosti poletov. 2008. № 11. S. 58-63.

7. Bukov V.N., Bocharov A.S., Sel'vesyuk N.I. Sintez konturov upravleniya letatel'nogo apparata s garantirovannoi tochnost'yu // Giroskopiya i navigatsiya. 2006. № 3 (54). S. 86.

8. Kulifeev Yu.B., Afanas'ev Yu.N. Algoritm avtomaticheskogo privedeniya samoleta k zadannym vysote i skorosti gorizontal'nogo poleta // Mekhatronika, avtomatizatsiya, upravlenie. 2011. № 9. S.54-58.

9. Makarenko V.G., Podorozhnyak A.A., Rudakov S.V., Bogomolov A.V. Inertsial'no-sputnikovaya navigatsionnaya sistema upravleniya transportnymi sredstvami // Problemy upravleniya. 2007. № 1. S. 64-71.

10. Savchenko A.Yu., Sel'vesyuk N.I. Razrabotka metodicheskogo apparata sinteza robastnykh detsentralizovannykh zakonov upravleniya s model'noi koordinatsiei // Informatika i sistemy upravleniya. 2014. № 2 (40). S. 158-167.

11. Gulevich S.P., Veselov Yu.G. Metodika opredeleniya koeffitsienta momenta tangazha bespilotnykh letatel'nykh apparatov po materialam naturnykh eksperimentov // Problemy bezopasnosti poletov. 2009. № 11. S. 47-52. 
12. Khar'kov V.P., Mikhalev I.S. Adaptivnyi algoritm upravleniya skorost'yu poleta letatel'nogo apparata // Informatsionno-izmeritel'nye i upravlyayushchie sistemy. 2011. T. 9. № 1. S. 9-15.

13. Kulifeev Yu.B. Opredelenie diskretnykh matematicheskikh modelei dinamicheskikh ob"ektov, zadannykh lineinymi differentsial'nymi uravneniyami s postoyannymi koeffitsientami // Informatsionno-izmeritel'nye i upravlyayushchie sistemy. 2008. T. 6. № 11. S. 5-19.

14. Kulifeev Yu.B., Likhachev I.V. Sintez algoritma upravleniya beskontaktnym ventil'nym dvigatelem metodom obratnykh zadach dinamiki // Mekhatronika, avtomatizatsiya, upravlenie. 2007. № 1. S. 28-36.

15. Khar'kov V.P., Korsun 0.H., Grebnev 0.H. Sintez upravleniya bespilotnym letatel'nym apparatom na osnove metoda obratnykh zadach dinamiki i parametricheskoi identifikatsii // Vestnik komp'yuternykh i informatsionnykh tekhnologii. 2010. № 12. S. 18-23.

16. Kulifeev Yu.B., Afanas'ev Yu.N. Algoritmizatsiya avtomaticheskoi posadki samoleta v usloviyakh meteominimuma // Mekhatronika, avtomatizatsiya, upravlenie. 2012. № 11. S. 60-66.

17. Gulevich S.P., Veselov Yu.G. Metod opredeleniya skorosti prizemleniya bespilotnykh letatel'nykh apparatov na osnove rezul'tatov matematicheskogo modelirovaniya i naturnykh eksperimentov // Problemy bezopasnosti poletov. 2008. № 6. S. 33-37.

18. Makarenko V.G., Bogomolov A.V., Rudakov S.V., Podorozhnyak A.A. Tekhnologiya postroeniya inertsial'no-sputnikovoi navigatsionnoi sistemy upravleniya transportnymi sredstvami s neirosetevoi optimizatsiei sostava vektora izmerenii // Mekhatronika, avtomatizatsiya, upravlenie. 2007. № 1. S. 39-44.

19. Kulifeev Yu.B., Alekseev E.O. Algoritm otrabotki zadannoi traektorii dvizheniya samoleta po vzletno-posadochnoi polose // Mekhatronika, avtomatizatsiya, upravlenie. 2007. № 10. S. 73-78.

20. Khar'kov V.P. Sintez optimal'nogo upravlenie nelineinymi dinamicheskimi sistemami na osnove kontseptsii obratnykh zadach dinamiki // Trudy mezhdunarodnogo simpoziuma Nadezhnost'i kachestvo. 2012. T. 1. S. 491-492.

21. Gurevich 0.S. Gol'berg F.D., Selivanov 0.D. Integrirovannoe upravlenie silovoi ustanovkoi mnogorezhimnogo samoleta. M.: Mashinostroenie, 1994. 304 s.

22. Kolomiets L.V., Fedorov M.V., Bogomolov A.V., Merezhko A.N., Soldatov A.S., Esev A.A. Metod podderzhki prinyatiya reshenii po upravleniyu resursami pri ispytaniyakh aviatsionnoi tekhniki // Informatsionno-izmeritel'nye i upravlyayushchie sistemy. 2010. T. 8. № 5. S. 38-40.

23. Rudakov I.S., Rudakov S.V., Bogomolov A.V. Metodika identifikatsii vida zakona raspredeleniya parametrov pri provedeniya kontrolya sostoyaniya slozhnykh sistem // Informatsionno-izmeritel'nye i upravlyayushchie sistemy. 2007. T. 5. № 1. S. 66-72.

24. Eremin E.M., Russkin A.V. Sposob rascheta otsenki maksimal'no dopustimoi vzletnoi massy bespilotnogo letatel'nogo apparata na etape ego proektirovaniya // Oboronnyi kompleks-nauchno-tekhnicheskomu progressu Rossii. 2014. № 1 (121). S. 39-42.

25. Baikin V.A., Stetsyuk A.N. Modelirovanie informatsionnogo upravleniya funktsionirovaniem sistemy kosmicheskoi svyazi i navigatsii // Trendy i upravlenie. - 2015. - 2. - C. 162 - 166. DOI: 10.7256/2307-9118.2015.2.14408. 